\title{
Gravitational instabilities induced by cluster environment? The encounter-induced angular momentum transfer in discs ${ }^{\star}$
}

\author{
S. Pfalzner and C. Olczak
}

\author{
I. Physikalisches Institut, University of Cologne, Zülpicher Str. 77, 50937 Cologne, Germany \\ e-mail: pfalzner@ph1.uni-koeln.de
}

Received 14 July 2006 / Accepted 18 September 2006

ABSTRACT

\begin{abstract}
Aims. The aim of this work is to understand to what extent gravitational interactions between the stars in high-density young stellar clusters, like the Orion Nebula Cluster (ONC), change the angular momentum in their protoplanetary discs.

Methods. Two types of simulations were combined $-N$-body simulations of the dynamics of the stars in the ONC, and angular momentum loss results from simulations of star-disc encounters.

Results. It is shown that in a star-disc encounter, the angular momentum loss is usually larger than the mass loss, so that the disc remnant has a lower specific angular momentum. Assuming an age of 1-2 Myr for the ONC, the disc angular momentum in the higher density region of the Trapezium is reduced by $15-20 \%$ on average. Encounters therefore play an important part in the angular momentum transport in these central regions, but are not the dominant process. More importantly, even in the outer cluster regions the angular momentum loss is on average 3-5\%. Here it is shown that a 3-5\% loss in angular momentum might be enough to trigger gravitational instabilities even in low-mass discs - a possible prerequisite for the formation of planetary systems.
\end{abstract}

Key words. galaxies: star clusters - planetary systems: protoplanetary disks - circumstellar matter - methods: N-body simulations planetary systems: formation

\section{Introduction}

Discs are seen as a prerequisite for the formation of planetary systems. There is increasing observational evidence that most, if not all, stars are initially surrounded by discs. For example, Lada et al. (2000) found that $80-85 \%$ of all stars in the Trapezium are surrounded by discs.

One visually compelling feature in the numerical study of these discs is the development of spiral arms either in self-gravitating high-mass discs or as an effect of encounters (Pfalzner 2003). In a few cases where discs have been observed with a sufficiently high spatial resolution, the existence of such spiral structures have been observationally confirmed (Clampin et al. 2003; Corder et al. 2005; Lin et al. 2006, for example). Spiral arms are always connected to mass and angular momentum transport. Our study of the importance of the encounterinduced angular momentum transport in a cluster environment has two motivations: angular momentum transport (a) in the late stages of accretion in star formation and (b) in spiral arm formation as a prerequisite for gravitational instabilities and fragmentation in planet formation scenarios.

The former is connected to the problem that the typical observed angular momentum of the cloud cores is so large (Bodenheimer 1995) that the star would be spinning at velocities higher than its break-up velocity if no means for angular momentum loss were present. One way out is the angular momentum transfer to the disc and then from the inner disc regions outwards. Many different mechanisms have been proposed for the latter (see Larson 2002, and references therein). Although often discarded as probably unimportant, encounter-induced angular

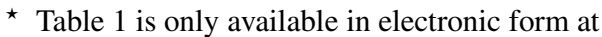
http: //www . aanda.org momentum transport is still often cited as possible mechanism. To clarify this point, we study disc angular momentum loss in a cluster environment in a quantitative, systematic manner for the first time. The second aim of this investigation is to achieve a better understanding of how the cluster environment influences the development of spiral structures in the discs through encountertriggered angular momentum transport. This might be of special importance as such spiral structures are a prerequisite in the theory of planet formation through gravitational instabilities.

The Orion Nebula Cluster (ONC) was chosen as the model cluster for these investigations because it is one of the beststudied regions in our galaxy, and observational constraints significantly reduce the modelling parameters. In addition, its high density suggests that stellar encounters might be relevant for the evolution of circumstellar discs. This is supported by recent investigations by Olczak et al. (2006) indicating that the effect of encounters has - at least for the disc mass loss - previously been underestimated.

The investigation involves three steps $-\mathrm{i}$ ) the determination of the encounter parameters in the ONC dynamics; ii) the angular momentum loss in isolated encounters; and iii) the combination of both results to investigate the angular momentum loss of the discs in the cluster. For the first step we use the cluster simulations described in Olczak et al. (2006) and star-disc simulations in Pfalzner et al. (2006) to determine a parameter list for the encounters for a model ONC in equilibrium and expansion, i.e., virial ratios of $Q_{\text {vir }}=0.5$ and $Q_{\text {vir }}=1$. The results are summarised in Sect. 2. This is followed by an investigation of the angular momentum loss in star-disc encounters in Sect. 3. There have been earlier investigations that looked at angular momentum transport in isolated star-disc encounters (Ostriker 1994; Larwood 1997; Hall et al. 1996; Pfalzner 2004) 
predominantly in prograde, coplanar encounters for low-mass discs covering only a small parameter range. Under these limitations simple $N$-body simulations suffice, and hydrodynamic effects and self-gravity within the disc can be largely neglected. Following this approach one has to keep in mind that the results have to be considered as upper limits (see discussion in Olczak et al. 2006, for the disc mass loss). We extended the parameter range covered in Pfalzner et al. (2005a) to larger mass ratios between the interacting stars and penetrating encounters to cover all situations necessary when modelling the ONC. In this work we neglect photo-evaporation as disc dispersal mechanism (Scally \& Clarke 2001), but this should be included in a future investigation. In Sect. 4 the results of Sects. 2 and 3 are combined to determine the disc angular momentum as a function of time. In Sect. 5 it is shown how the angular momentum mass loss is influenced by the assumptions made and a discussion is given of how this angular momentum loss can trigger gravitational instabilities.

\section{Model and cluster simulations}

We combine simulations of the dynamics of the ONC determining the interaction parameters of close encounters between stars in the cluster (Olczak et al. 2006) and with results of isolated star-disc encounter simulations described in Pfalzner et al. (2006). In contrast to these previous studies, the emphasis in the current investigation is not on the mass but, the angular momentum loss of the discs. The dynamical model of the ONC presented here contains only stellar components, neglecting gas and the potential of the background molecular cloud OMC 1. The cluster models were set up with a spherical density distribution $\rho(r) \propto r^{-2}$, by randomly generating the masses according to the mass function given by Kroupa et al. (1993) in a range $50 M_{\odot} \geq M^{*} \geq 0.08 M_{\odot} . \theta^{1} \mathrm{C}$ Ori was directly assigned a mass of $50 M_{\odot}$ and placed at the cluster centre. The velocity distribution of the stars was assumed to be Maxwellian.

The ONC cluster simulations were performed with NBODY6 ${ }^{++}$(Spurzem 1999) and modelled for $13 \mathrm{Myr}$ - the assumed lifetime of $\theta^{1} \mathrm{C}$ Ori. The quality of the dynamical models was judged by comparing them to observational data at 1-2 Myr, marking the range of the mean ONC age. For more details of the selection process, see Olczak et al. (2006). The quantities of interest were: number of stars, half-mass radius, number densities, velocity dispersion and projected density profile. Here we mainly describe the case of the $\mathrm{ONC}$ being in virial equilibrium; in Sect. 4 the changes one can expect for an expanding cluster will be briefly considered.

Assuming that only two-body encounters occur and that higher-order encounters are negligible, the effect of an encounter can be investigated by considering it to be isolated from the rest of the cluster. During the course of the simulation the information of all perturbing events of each stellar disc, i.e., both masses, the relative velocity and the eccentricity are recorded. However, later on the latter is not applied as such. To limit the parameter space only the angular momentum loss of parabolic encounters is used. The eccentricity values are utilised to estimate the error introduced by such a simplification.

\section{Angular momentum loss in star-disc encounters}

This part of the investigation is mainly based on the earlier work by Pfalzner et al. (2005a), where a parameter study was performed for a star of mass $M_{1}^{*}=1 M_{\odot}$ surrounded by a disc

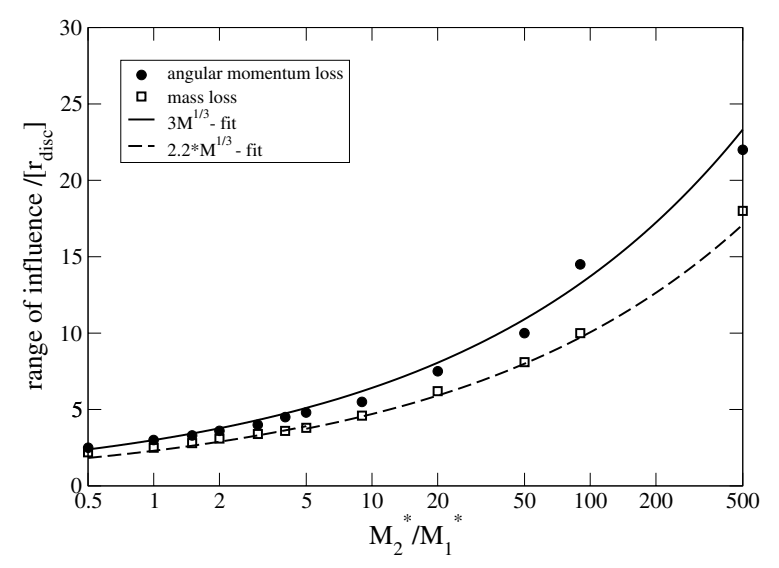

Fig. 1. Interaction range for mass and $A M L$ as a function of the mass ratio of the interacting stars in units of the disc radius.

perturbed by the fly-by of a second star of mass $M_{2}^{*}$. When simulating isolated encounter events, the observational evidence that most discs in the ONC are of low mass $m_{\mathrm{d}}$, i.e., $m_{\mathrm{d}} / M^{*} \ll$ 0.1 , reduces the complexity of the numerical approach in several ways: low-mass discs do not significantly influence the encounter orbit, self-gravitation can be neglected, and the results are scalable to other star masses. The effect of viscosity is negligible in this case, too, as the viscous timescale is so much longer than the timescale of the encounter. Another simplification is that only coplanar, prograde encounters were investigated. According to studies on inclined and retrograde star-disc encounters (Ostriker 1994; Heller 1995; Hall et al. 1996; Pfalzner et al. 2005a), due to these approximations the results can only be interpreted as an upper limit for the angular momentum loss. We will dicuss this in more detail at the end of this section.

The disc is assumed to extend to $r_{\mathrm{d}}=100 \mathrm{AU}$ and the surface density initially has a $1 / r$-dependence. The angular momentum loss induced by the fly-by of a star on such a star-disc system depends on various parameters specific to the stars and discs involved and the encounter orbit. The above-mentioned earlier study (Pfalzner et al. 2005a) was limited to non-penetrating encounters with star mass ratios in the interval $0.1<M_{2}^{*} / M_{1}^{*}<5$. Here, the parameter study is extended to the higher mass ratios of up to $M_{2}^{*} / M_{1}^{*}=50.0 M_{\odot} / 0.08 M_{\odot}$, which occur in the ONC simulations. The additional fractional angular momentum loss (AML) values can be found in Table 1 available in the electronic version of this article. The improved fit function for $(\Delta J / J)_{\text {total }}$ is

$$
\begin{aligned}
\left(\frac{\Delta J}{J}\right)_{\text {total }}= & 1.02\left(\frac{M_{2}^{*}}{M_{1}^{*}+M_{2}^{*}}\right)^{0.5 r_{\mathrm{p}}} \\
& \times \exp \left[-\sqrt{\frac{M_{1}^{*}\left(r_{\mathrm{p}}-0.7 r_{\mathrm{p}}^{0.5}\right)^{3}}{M_{2}^{*}}}\right],
\end{aligned}
$$

where $r_{\mathrm{p}}=r_{\text {peri }} / r_{\text {disc }}$ is the periastron separation $r_{\text {peri }}$ in units of the disc radius $r_{\text {disc }}$.

The most important results for the cluster are that

1. the AML is always larger than the relative mass loss, and

2. the angular momentum of the disc is affected by much more distant encounters than the disc mass.

The latter is illustrated by Fig. 1, where the relative distance $r_{\text {peri }} / r_{\text {disc }}$ at which the angular momentum is changed by at least $3 \%$ (the average error value) is shown as a function of the mass 
a)
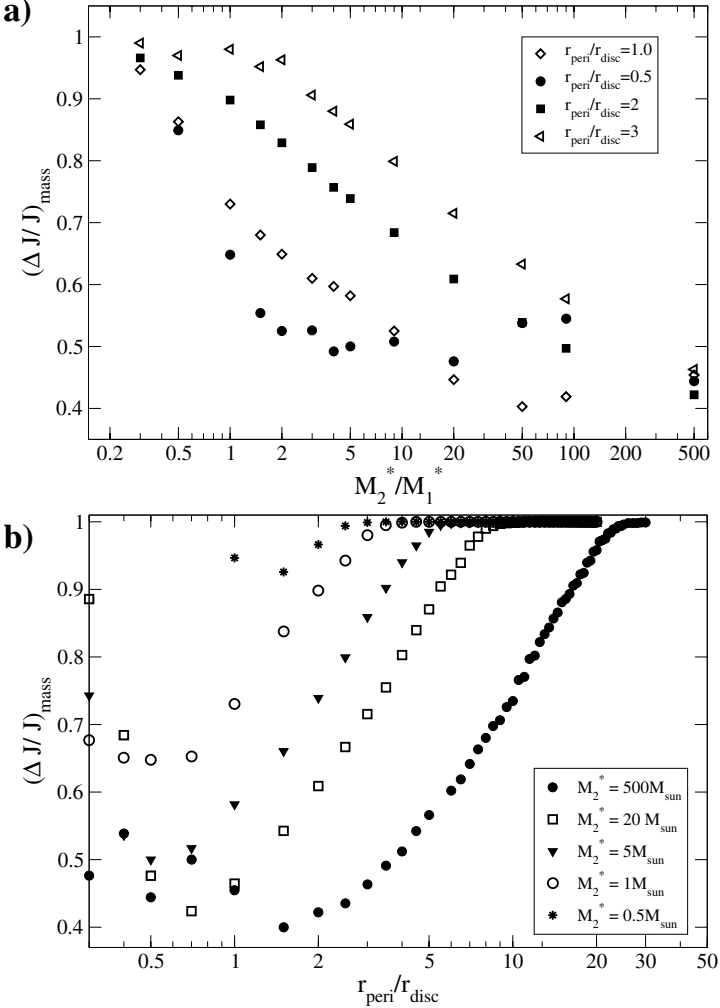

Fig. 2. Specific angular momentum after an coplanar, prograde parabolic encounter compared to that of the initial disc shown as a function of a) the perturber mass $M_{2}^{*}$, and b) the encounter periastron in units of the disc size.

ratio $M_{2}^{*} / M_{1}^{*}$. Given a sufficiently large perturber mass, even encounters as distant as 20 times the disc radius can reduce the angular momentum in the disc by $10 \%$ or more without changing the disc mass at all.

A considerable part of the angular momentum is carried away with the matter that becomes unbound. However, what one is really interested in is to what degree the angular momentum per particle is lower in the remaining disc - only this can eventually facilitate accretion of matter onto the star. A measure of this specific angular momentum can be defined by

$(\Delta J / J)_{\mathrm{mass}}:=\left(1-\frac{\Delta J}{J}\right) /\left(1-\frac{\Delta m}{m}\right)$.

Figures $2 \mathrm{a}$ and $2 \mathrm{~b}$ show the specific angular momentum obtained by using Table 1 in this paper and Table 3 in Olczak et al. (2006) as a function of $M_{2}^{*} / M_{1}^{*}$ and $r_{\mathrm{p}}$, respectively. ${ }^{1}$ For large mass ratios there exists a lower limit of 0.4 for $\left(J_{\text {enc }} / J\right)_{\text {mass }}$ for all considered periastra. This means that in a single encounter, however strong, the specific angular momentum can only be reduced to $\sim 40 \%$ of that in the initial disc. However, such a change of angular momentum is achieved over a wide range of interaction parameters, so that in repeated encounters the angular momentum of the remaining disc could nevertheless be considerably reduced.

Since the cluster consists of a wide spectrum of star masses, these simulation results obtained for $M_{1}^{*}=1 M_{\odot}$ are generalised

${ }^{1}$ Note, fit formula 1 here and Eq. (4) in Olczak et al. (2006) are not of high enough accuracy to determine $\left(J_{\text {enc }} / J\right)_{\text {mass }}$ in Eq. (2) in the entire parameter range required for the $\mathrm{ONC}$. a)

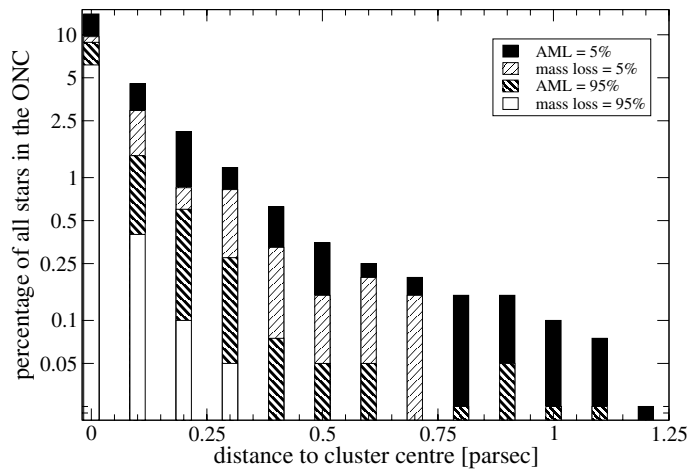

b)

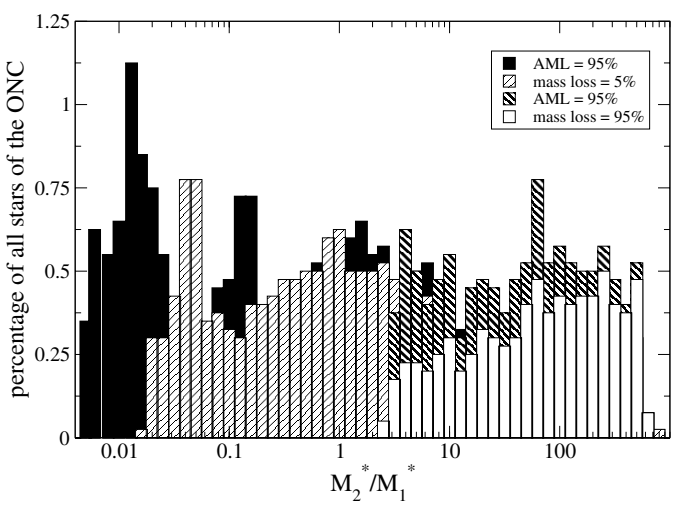

Fig. 3. a) Percentage of stars in bins with a 5\% (lowest considered bin) or $95 \%$ (highest considered bin) AML in the disc as a function of distance to the cluster centre at $2 \mathrm{Myr}$ averaged over 20 simulations; b) shows the same as a function of the mass ratio $M_{2}^{*} / M_{1}^{*}$ of the two interacting stars. For comparison, in both figures the same is shown for the mass loss, too.

by scaling the disc radius according to

$r_{\mathrm{disc}}=r_{\mathrm{disc}}\left(1 M_{\odot}\right) \sqrt{M_{1}^{*}\left[M_{\odot}\right]}$.

In the cluster simulations, $r_{\mathrm{disc}}\left(1 M_{\odot}\right)$ was assumed to be 150 AU. Scaling the disc size with the star mass seems intuitively right, but observations are not so unambiguous: Vicente \& Alves (2005) found a correlation between disc diameters and stellar masses using a sample of proplyds from Luhman et al. (2000), but not for the data from Hillenbrand (1997). However, the present Trapezium is probably not in its primordial state, as various disc destruction processes have most likely altered the disc sizes considerably.

As mentioned earlier, the encounter results summarised by Eq. (2), Fig. 2, and Table 1 represent upper limits to the angular momentum loss induced by encounters. The reason is that they are obtained for prograde, coplanar, and parabolic encounters only. In addition, it was assumed that only one of the stars was surrounded by a disc. The latter is somewhat contradicting - assuming that each star is initially surrounded by a disc, but at the same time considering an encounter with a discless star. There are two reasons why this was done: first, encounters where both stars are surrounded by discs are less well investigated, and second Pfalzner et al. (2005b) showed that the star-disc results can be generalised to disc-disc encounters as long as there is no mass exchange between the discs. In the case of a mass exchange, the discs can to some extent be replenished with material of very low angular momentum so that the specific angular momentum loss (SAML) would be underestimated. 

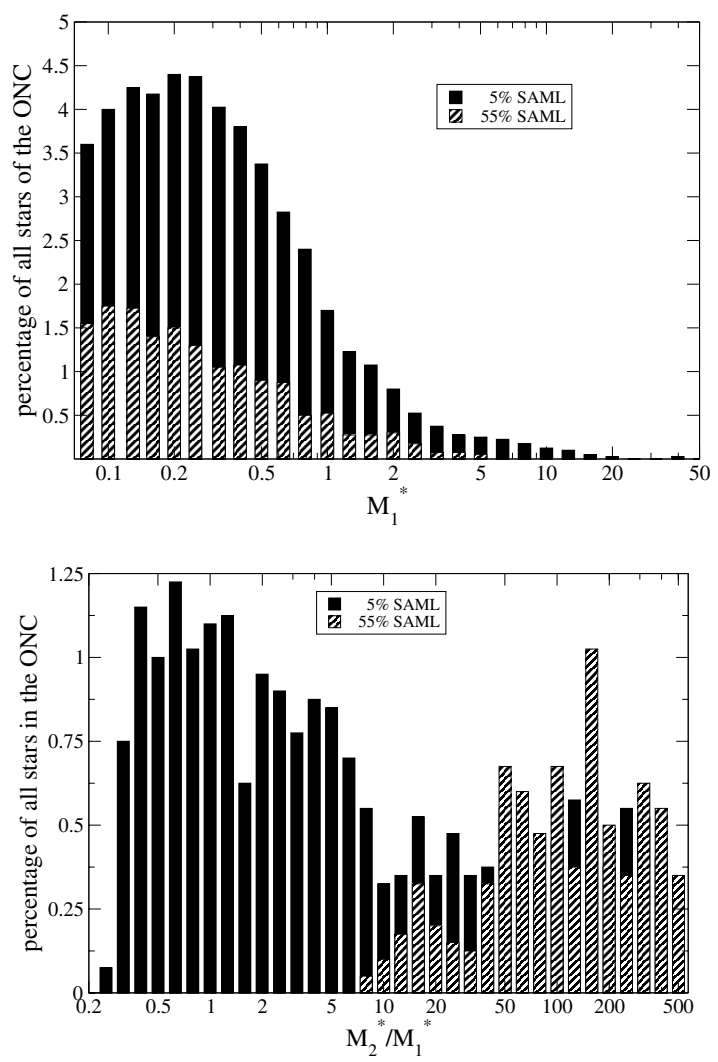

Fig. 4. Percentage of stars with a SAML as defined by Eq. (2) as function of the stellar mass averaged over 10 simulations. The bin for 5\% and now 55\% (as the highest obtainable SAML is $\sim 60 \%$ ) loss are shown.

Most encounters in the cluster simulations are not parabolic, but hyperbolic. In such hyperbolic encounters, the angular momentum loss is lower because the disturber is not long enough in the vicinity of the star-disc system to remove angular momentum in an efficient way. However, considering only the stars that lose more than $90 \%$ of their disc mass, the eccentricity distribution has a maximum at $\epsilon \approx 1$.

In a cluster that is not highly flattened, it seems rather unlikely that the encounter planes are aligned to a high degree. Therefore one would expect most encounters to be non-coplanar. Pfalzner et al. (2005a) showed that, as long as the inclination is not larger than $45^{\circ}$, the AML in the encounter is only slightly $(<10 \%)$ reduced in comparison to a coplanar encounter. If however the orientation is completely random and a $90^{\circ}$-encounter, the most likely encounter scenario, the mass loss could be significantly reduced, a point which would need further investigation.

It is often argued that consecutive encounters would not lead to any significant additional momentum transport because the outer edge of the disc is truncated by the first encounter - the part that is mainly involved in the interaction. However, Pfalzner et al. (2004) demonstrated that a second encounter can indeed lead to the same relative AML as in the first encounter. This implies that a succession of distant encounters might well be able to transport a higher amount of angular momentum outwards than previously thought (see Moeckel \& Bally 2006, too). In future studies, it should be investigated whether this holds for the entire parameter space covered here.

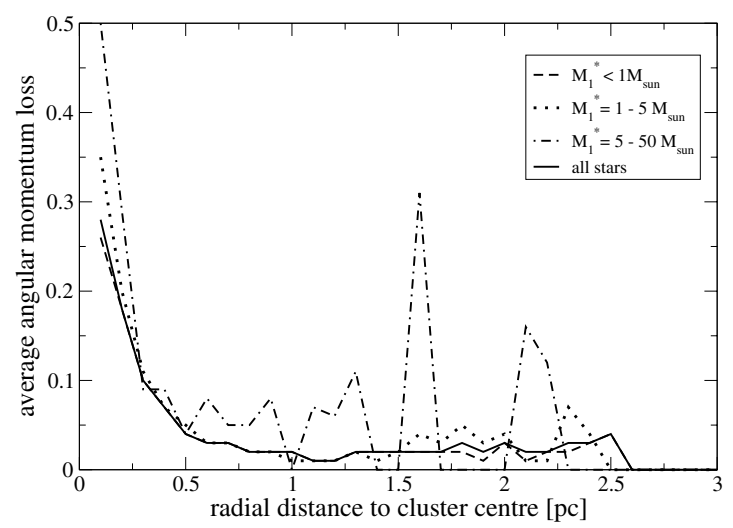

Fig. 5. SAML as a function of the radial distance to the cluster centre averaged over 20 simulations.

\section{Angular momentum loss in the ONC}

In the following the cluster results are combined with those of Sect. 3 to investigate the encounter-induced angular momentum loss of the discs in the ONC. First we determine the AML using Eq. (1). The fact that the angular momentum loss is usually larger than the mass loss is reflected by the cluster simulations in several ways: as Fig. 3a shows, the number of stars with, for example, more than $5 \%$ or $95 \%$ relative angular momentum loss in the disc is somewhat higher than the number of discs with a more than $5 \%$ or $95 \%$ mass loss. Due to the lower stellar number density in the outer cluster regions, both the mass loss and the AML decrease with distance from the cluster centre. However, the AML extends to more distant cluster regions - for the $5 \%$ case the mass loss extends to $\sim 0.75 \mathrm{pc}$, whereas the AML reaches to $\sim 1.25 \mathrm{pc}$. For high losses (95\%), the mass loss extends only to $0.3-0.4 \mathrm{pc}$, but the angular momentum to $\sim 1.1$ pc. Figure $3 \mathrm{~b}$ demonstrates that lower relative perturber masses $M_{2}^{*} / M_{1}^{*}$ are required to remove at least $5 \%$ of the angular momentum than to carry off the same percentage of disc mass.

The dependence on the distance from the cluster centre of the SAML is very similar to that of the AML, i.e. beyond $0.3 \mathrm{pc}$ no mass loss occurs, and AML and SAML happen as far out as $\sim 1.25$ pc. However, some differences nevertheless occur. They are due to the fact most massive stars have basically lost all their mass and are not considered for this mass-corrected value anymore. In addition, the dependence on $M_{2}^{*} / M_{1}^{*}$ is moved to higher $M_{2}^{*} / M_{1}^{*}$-values (see Fig. 4b). This might indicate that lowmass encounters can remove only mass with angular momentum, whereas high-mass encounters can do the opposite.

Figure 5 shows that the SAML is reduced most in the central region of the cluster (by $\sim 15-20 \%$ ), but throughout the cluster there is SAML even if only on a $3-5 \%$ per cent level in the outer cluster areas. As mentioned in Sect. 3, it is not clear what the primordial disc-size distribution in a cluster is. If we assume that the disc size does not scale with the disc mass, but assume initially equal disc sizes (150 AU), the SAML is larger at all distances (see Fig. 6). This is most pronounced in the Trapezium $(\sim 30-40 \%)$, but even further outside the SAML increases by $\sim 1 \%$. The reason is that the majority of stars have masses $<1 M_{\odot}$. Since an equal disc size for all stars is equivalent to a larger disc size for them, this results in a higher SAML.

As the velocity dispersion of the ONC is not well determined, the next point to investigate is how the SAML changes if we consider the cluster to be expanding. Testing the $Q_{\text {vir }}=1$ case, the angular momentum reductions turns out to be larger 


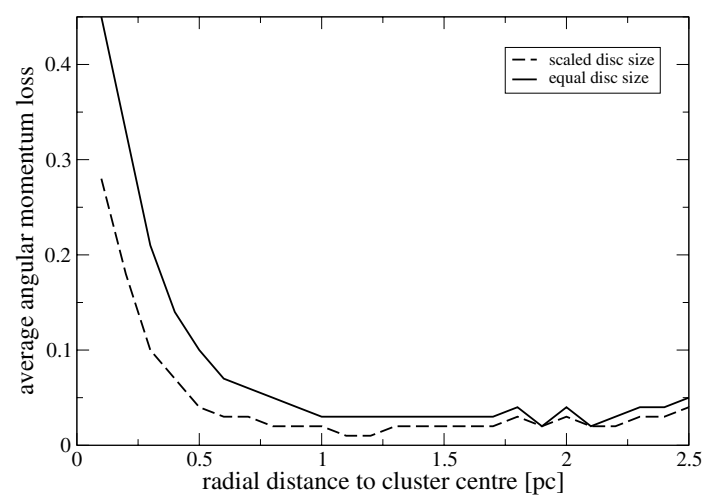

Fig. 6. SAML for equal and scaled disc size as a function of the radial distance to the cluster centre.

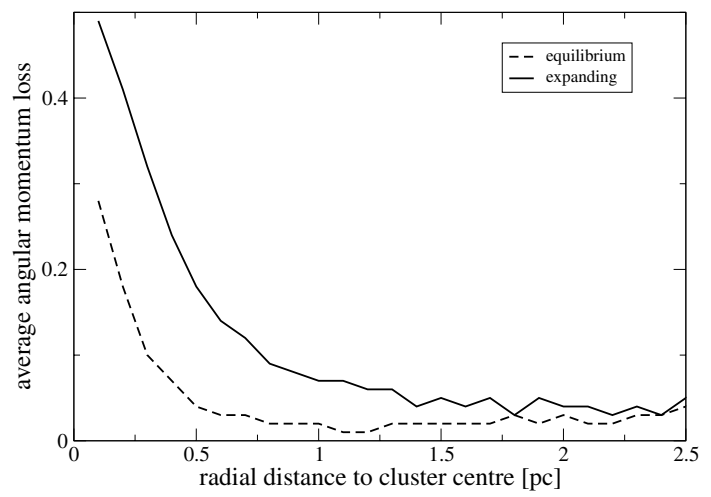

Fig. 7. SAML as function of radial distance to cluster centre. Comparison between cluster in equilibrium and an expanding cluster for scaled disc size.

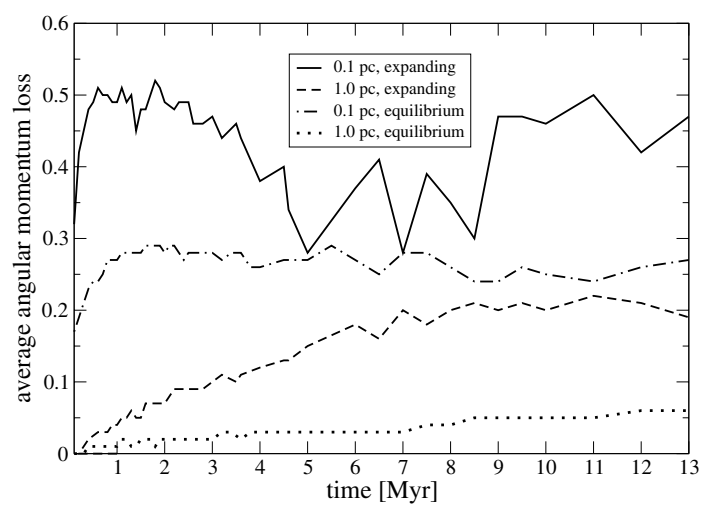

Fig. 8. SAML as function of time for an expanding cluster and a cluster in equilibrium at $0.1 \mathrm{pc}$ and $1.0 \mathrm{pc}$.

in the entire $\mathrm{ONC}$ with the largest differences in the Trapezium region (see Fig 7). Figure 8 shows that the difference in SAML happens early on in the clustre development. Here the density in the expanding cluster is initially higher in the center leading to more and stronger encounters.

\section{Discussion and conclusions}

Combining simulations that follow the path of the stars in the cluster with angular momentum loss results obtained from the simulations of isolated encounters, the encounter-induced angular momentum loss has been investigated for the ONC. The main outcome of these simulations is that for most stars in the $\mathrm{ONC}$, encounters reduce the angular momentum on average only by $3-5 \%$ in the remaining disc compared to the initial disc. However, this kind of reduction happens even in the outer parts of the ONC, so that the disc angular momentum is affected by encounters throughout the entire cluster. If one considers only the central dense Trapezium region, the SAML increases to the $10-30 \%$ level. This means that although encounters in the cluster environment do lead to angular momentum loss in the disc, it is not the predominant process for angular momentum transfer.

However, it would be premature to declare encounters as unimportant for the disc development. On the contrary, encounter-induced angular momentum transport may still play an important part in the disc evolution. As one can see in Fig. 9, an SAML in the 3-5\% regime, which we showed to be present in the entire $\mathrm{ONC}$, is sufficient to trigger pronounced spiral arm structures in the disc. As one can see, similar structures appear independent of the mass of the perturber given the same angular momentum change.

As mentioned above, such spiral structures have been detected in the few cases where discs have been observed with a sufficiently high spatial resolution (for example Clampin et al. 2003; Corder et al. 2005; Lin et al. 2006). A fly-by has often been rejected as a possible cause of the spiral pattern because no stars have been found in the immediate vicinity of the star-disc system. However, as the results in Sect. 3 show, angular momentum change can be induced by relatively distant encounters given that the mass ratio between the star and the perturber is large. For example, for $M_{2}^{*} / M_{1}^{*}=50$ an encounter with a periastron $\sim 14$ times the disc radius could create a spiral arm pattern similar to those shown in Fig. 9. Considering the far range of interaction in many cases, a much larger area would have to be considered in a search for possible encounter partners.

What consequences can such spiral patterns in the disc have? The spiral arm pattern above might be the starting point for gravitational instabilities (in the above simulations, self-gravity of the disc is not considered, so clumping cannot occur). So far gravitational instabilities have predominantly been investigated for isolated high-mass discs (for a summary, see Durisen et al. 2006). In this context gravitational instabilities can produce global spiral arms and disc fragmentation into dense clumps and substructures. Possibly these dense clumps may become precursors to gas giant planets (Kuiper 1951; Cameron 1978; Boss 1998).

Modern numerical simulations, beginning with Papaloizou $\&$ Savonije (1991), show that non-axisymmetric disturbances become unstable for $Q=c_{\mathrm{s}} \kappa / \pi G \Sigma<1.5$, where $c_{\mathrm{s}}$ is the sound speed, $\kappa$ the epicyclic frequency, $\mathrm{G}$ the gravitational constant, and $\Sigma$ the surface density. When the gravitational instabilities emerge from the linear regime, they may either saturate at nonlinear amplitude or fragment the disc (Durisen et al. 2006). What happens depends on whether a balance can be reached between heating and the loss of disk thermal energy by radiative or convective cooling. Although there is agreement on conditions for fragmentation, it is an open question whether real discs cool fast enough for fragmentation to occur and whether these fragments last long enough to contract into permanent protoplanets (Durisen et al. 2006).

A prerequisite for the gravitational instabilities in isolated discs is a high enough disc mass that self-gravity can carry small perturbations to fragmentation in the non-linear regime. However, observations show that high-mass discs seem to be the exception rather than the rule. Various mechanisms have been suggested for triggering gravitational instabilities, among them, the formation of massive discs from the collapse of protostars (Laughlin \& Bodenheimer 1994; Yorke \& Bodenheimer 1999; 


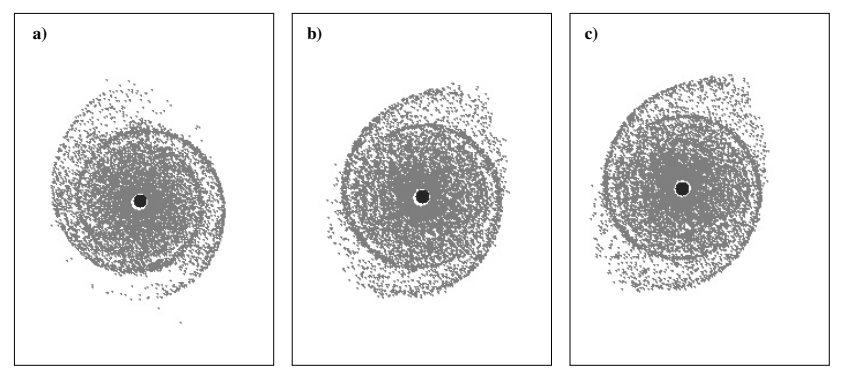

Fig. 9. Examples of encounters with a (SAML) of 3-5\%. a) shows an encounter where $M_{2}^{*}=0.1 M_{\odot}$ and $\left.r_{\mathrm{p}}=2.0, \mathbf{b}\right)$ an encounter where $M_{2}^{*}=$ $1 M_{\odot}$ and $r_{\mathrm{p}}=3.5$, and c) an encounter where $M_{2}^{*}=5 M_{\odot}$ and $r_{\mathrm{p}}=5.5$.
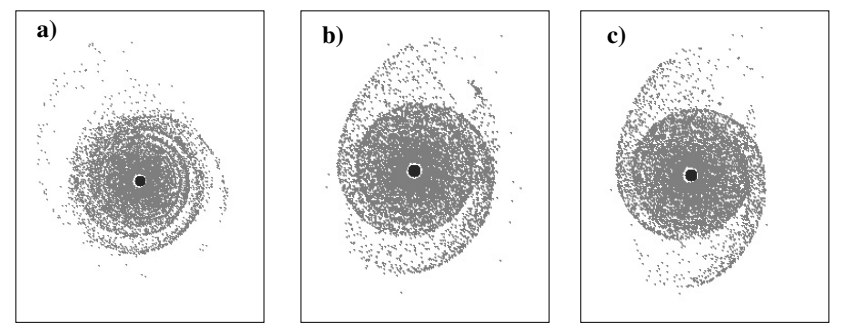

Fig. 10. Examples of encounters with a SAML of $\sim 20 \%$. a) shows an encounter where $M_{2}^{*}=0.1 M_{\odot}$ and $\left.r_{\mathrm{p}}=1.5, \mathbf{b}\right)$ an encounter where $M_{2}^{*}=1 M_{\odot}$ and $r_{\mathrm{p}}=2.8$, and c) an encounter where $M_{2}^{*}=5 M_{\odot}$ and $r_{\mathrm{p}}=4.5$.

Boss 1998), clumpy infall onto the disc (Boss 1998), cooling from stable to unstable state, perturbation by binary companions (Mayer et al. 2005), and close encounters with star/disc systems (Boffin et al. 1998; Lin et al. 1998).

Investigating binary systems, Mayer et al. (2005) found that the degree of fragmentation depends on the mass of the disc and the cooling rate. The simulations by Mayer et al. (2005) and Boss (2006) both indicate that apparently, in contrast to isolated discs, in binary systems lighter discs are more likely to fragment. This counterintuitive result reflects the fact that intense heating from spiral shocks can suppress the formation of permanent clumps. A balance between compression/shock heating and cooling is required for fragmentation.

The situation in an encounter is similar to that in a binary system because the secondary star induces a non-axisymmetric perturbation onto the star-disc system, but with the difference that the perturbation is not recurring. At the moment it is unclear whether encounters enhance disc fragmentation or not. Investigations by Lodatao et al. (2006) have found that discs with long cooling timescales are actually rendered more stable by encounters. On the other hand, Shen \& Wadsley (2006) do get encounter induced fragmentation when they use an isothermal equation of state. In the future comprehensive investigations are required to determine by detailed simulations with the right radiation hydrodynamics which amount of angular momentum change is needed to obtain stable clumps - is it the stronger interactions (10-30\% angular momentum change) that we find in the clustre center or rather the weaker (3-5\% angular momentum change) ones further outside?

There is actually a second means by which spiral structures induced by encounters could support planet formation. In the classical picture of planet formation (for a summary see Weidenschilling 1993) sticking of dust grains and further agglomeration eventually leads to planet formation. Rice et al. (2006) showed that dust can accumulated in spiral shocks induced in self-gravitating disc. If this happens in the same fashion in encounter induced spirals, encounters could trigger planet formation via dust agglomeration as well. The higher dust density and possibly the higher velocity dispersion in the spiral arm could assist dust agglomeration. However, the timescale of the lifetime of spiral structures is typically only 1000 to 2000 years, which would be very stringent for this planet formation scenario.

From the simulations in this paper we have shown that in systems like the ONC such an encounter situation is a common event throughout the cluster. Therefore, if planets can be formed through gravitational instabilities at all, encounters in a cluster environment are a likely trigger for their occurance.

Acknowledgements. We are grateful to R. Spurzem for providing the Nbody6++ code for the cluster simulations and want to thank the referee for the very helpful comments. Simulations were partly performed at the John von Neumann Institute for Computing, Research Center Jülich, Project HKU14.

\section{References}

Aarseth, S. 2003, Gravitational N-Body Simulations (Cambridge: CUP)

Adams F. C., \& Lin, D. N. C. 1993, in Protostars and Planets III, ed. E. H. Levy,

\& J. I. Lunine (Tuscon: Univ. of Arizona Press), 721

Bodenheimer, P. 1995, ARA\&A, 33, 199

Boffin, H. M. J., Watkins, S. J., Bhattal, A. S., Francis, N., \& Whitworth, A. P. 1998, MNRAS, 300, 1189

Boss, A. P. 1998, ApJ, 503, 923

Boss, A. P. 2006, ApJ, 641, 1148

Cameron, A. G. 1978, Moon Planets, 18, 5

Clampin, M., Krist, J. E., \& Ardila, D. R. 2003, AJ, 126, 385

Corder, S., Eisner, J., \& Sargent, A. 2005, ApJ, 622, L133

Clarke, C. J., \& Pringle, J. E. 1993, MNRAS, 261, 190

Durison, R. H., Boss, A. P., Mayer, L., et al. 2006, [arXiv: astro-ph/603179]

Gammie, C. F. 2001, ApJ, 553, 174

Hall, S. M., Clarke, C. J., \& Pringle, J. E. 1996, MNRAS, 278, 303

Heller, C. H. 1993, ApJ, 408, 337

Heller, C. H. 1995, ApJ, 455, 252

Hillenbrand, L. A. 1997, AJ, 113, 1733

Kroupa, P., Tout, C. A., \& Gilmore, G. 1993, MNRAS, 262, 545

Kuiper, G. P. 1951, in Proceedings of a Topical Symposium, ed. J. A. Hynek (New York: McGraw-Hill), 357

Lada, C. J., Muench, A. A., Haisch, K. E. et al. 2000, AJ, 120, 3162

Larson, R. B. 2002, MNRAS, 332, 155

Nelson, R. P., Papaloizou, J. C. B., Larwood, J. D., \& Terquem, C. 1997, in Accretion Disks - New Aspects, Proceedings of the EARA Workshop, ed. H. Meyer-Hofmeister (Heidelberg: Springer) 182,

Laughlin, G., \& Bodenheimer P. 1994, ApJ, 435, 335

Lin, D. N. C., Laughlin, G., Bodenheimer, P., \& Roczyczka, M. 1998, Science, 281,2025

Lin, S.-Y., Ohashi, N., Lim, J., et al. 2006, [arXiv: astro-ph/0603414]

Lodato, G., Meru, F., Clarke, C., \& Rice, W. K. M., MNRAS, submitted

Luhman, K. L., Rieke, G. H., Young, E. T., et al. 2000, ApJ, 540, 1016

Mayer, L., Wadsley, J., Quinn, T., \& Stadel, J. 2005, MNRAS, 363, 641

McCaughrean, M., Zinnecker, H., Andersen, M., Meeus, G., \& Lodieu, N. 2002, The Messenger, 109, 28

Moeckel, N., \& Bally, J. 2006, [arXiv: astro-ph/0608070]

Olczak, C., Pfalzner, S., \& Spurzem, R. 2006, ApJ, 642, 1140

Ostriker, E. C. 1994, ApJ, 424, 292

Papaloizou, J. C. B., \& Savonije 1995, MNRAS, 248, 353

Pfalzner, S. 2003, ApJ, 592, 986

Pfalzner, S. 2004, ApJ, 602, 356

Pfalzner, S., Vogel, P., Scharwächter, J., \& Olczak, C. 2005a, A\&A, 437, 976

Pfalzner, S., Umbreit, S., \& Henning, Th. 2005b, ApJ, 629, 526

Pfalzner, S., Olczak, C., \& Eckart, A. 2006, A\&A, 454, 811

Rice, W. K. M., Lodato, G., Pringle, J. E., Armitage, P. J., \& Bonnell, I. A. 2006, [arXiv: astro-ph/0607268]

Scally, A., \& Clarke, C. 2001, MNRAS, 325, 449

Shen, S., \& Wadsley, J. 2006, [arXiv: astro-ph/0608325]

Spitzer, L., \& F. H. 1968, in Nebulae and Interstellar Matter, ed. B. M. Middlehurst, \& L. H. Aller (Univ. of Chicago Press), 1

Spurzem, R. 1999, Comp. Astroph., 109, 407

Vicente, S. M., \& Alves, J. 2005, A\&A, 441, 195

Weidenschilling, S. J., \& Cuzzi, J. N., 1993, in Protostars and Planets III, ed. E. H. Levy, \& J. I. Lunine, 1031

Yorke, H. W., \& Bodenheimer, P. 1999, ApJ, 525, 330 


\section{Online Material}


S. Pfalzner and C. Olczak: Cluster as trigger for gravitational instabilities, Online Material p 2

Table 1. Table of AML $\left(\Delta J_{\mathrm{d}} / J_{\mathrm{d}}\right)_{\text {bound }}$ for all simulated configurations of parabolic etscape.com/ $(e=1)$ star-disc encounters. The first row contains the relative perturber masses $M_{2}^{*} / M_{1}^{*}$, the first column contains the relative periastra $r_{\mathrm{p}} / r_{\mathrm{d}}$. Results from simulations are denoted by four digits, the values " 0.0 " were edited manually.

\begin{tabular}{|c|c|c|c|c|c|c|c|c|c|c|c|c|c|c|}
\hline & 500.0 & 90.0 & 50.0 & 20.0 & $\overline{9.0}$ & 5.0 & 4.0 & 3.0 & 2.0 & 1.5 & 1.0 & 0.5 & 0.3 & 0.1 \\
\hline 0.1 & 0.949 & 0.913 & 0.937 & 0.911 & 0.873 & 0.797 & 0.713 & 0.747 & 0.736 & 0.708 & 0.688 & 0.633 & 0.488 & 0.239 \\
\hline 0.2 & 0.969 & 0.941 & 0.947 & 0.952 & 0.938 & 0.900 & 0.863 & 0.866 & 0.860 & 0.826 & 0.719 & 0.592 & 0.391 & 0.212 \\
\hline 0.3 & 0.990 & 0.991 & 0.986 & 0.969 & 0.959 & 0.948 & 0.934 & 0.908 & 0.882 & 0.855 & 0.797 & 0.598 & 0.310 & 0.190 \\
\hline 0.4 & 0.993 & 0.993 & 0.993 & 0.987 & 0.981 & 0.963 & 0.953 & 0.935 & 0.891 & 0.897 & 0.784 & 0.577 & 0.238 & 0.170 \\
\hline 0.5 & 0.996 & 0.994 & 0.993 & 0.992 & 0.970 & 0.949 & 0.941 & 0.919 & 0.894 & 0.856 & 0.783 & 0.554 & 0.201 & 0.164 \\
\hline 0.7 & 0.995 & 0.994 & 0.993 & 0.975 & 0.945 & 0.908 & 0.894 & 0.864 & 0.823 & 0.792 & 0.726 & 0.533 & 0.186 & 0.143 \\
\hline 1.0 & 0.995 & 0.987 & 0.975 & 0.941 & 0.885 & 0.822 & 0.797 & 0.765 & 0.708 & 0.660 & 0.586 & 0.382 & 0.219 & 0.126 \\
\hline 1.5 & 0.992 & 0.955 & 0.926 & 0.860 & 0.762 & 0.681 & 0.642 & 0.587 & 0.501 & 0.447 & 0.340 & 0.200 & 0.152 & 0.048 \\
\hline 2.0 & 0.981 & 0.914 & 0.870 & 0.768 & 0.637 & 0.521 & 0.476 & 0.407 & 0.316 & 0.249 & 0.182 & 0.097 & 0.052 & 0.003 \\
\hline 2.5 & 0.963 & 0.865 & 0.805 & 0.662 & 0.508 & 0.375 & 0.321 & 0.263 & 0.187 & 0.136 & 0.079 & 0.021 & 0.006 & 0.000 \\
\hline 3.0 & 0.943 & 0.810 & 0.728 & 0.560 & 0.380 & 0.239 & 0.198 & 0.143 & 0.089 & 0.060 & 0.020 & 0.003 & 0.001 & 0.000 \\
\hline 3.5 & 0.917 & 0.755 & 0.657 & 0.461 & 0.267 & 0.152 & 0.118 & 0.079 & 0.032 & 0.013 & 0.005 & 0.000 & 0.000 & 0.000 \\
\hline 4.0 & 0.894 & 0.691 & 0.575 & 0.365 & 0.191 & 0.077 & 0.050 & 0.024 & 0.007 & 0.003 & 0.001 & 0.000 & 0.000 & 0.000 \\
\hline 4.5 & 0.859 & 0.632 & 0.500 & 0.272 & 0.109 & 0.037 & 0.020 & 0.008 & 0.002 & 0.001 & 0.000 & 0.000 & 0.000 & 0.000 \\
\hline 5.0 & 0.833 & 0.563 & 0.422 & 0.214 & 0.070 & 0.015 & 0.001 & 0.002 & 0.001 & 0.000 & 0.000 & 0.000 & 0.000 & 0.000 \\
\hline 5.5 & 0.797 & 0.493 & 0.352 & 0.139 & 0.003 & 0.005 & 0.000 & 0.001 & 0.000 & 0.000 & 0.0 & 0.0 & 0.0 & 0.0 \\
\hline 6.0 & 0.770 & 0.434 & 0.297 & 0.105 & 0.014 & 0.002 & 0.000 & 0.000 & 0.000 & 0.000 & 0.0 & 0.0 & 0.0 & 0.0 \\
\hline 6.5 & 0.737 & 0.373 & 0.231 & 0.072 & 0.007 & 0.001 & 0.0 & 0.000 & 0.0 & 0.0 & 0.0 & 0.0 & 0.0 & 0.0 \\
\hline 7.0 & 0.703 & 0.313 & 0.198 & 0.036 & 0.003 & 0.001 & 0.0 & 0.000 & 0.0 & 0.0 & 0.0 & 0.0 & 0.0 & 0.0 \\
\hline 7.5 & 0.663 & 0.298 & 0.140 & 0.022 & 0.001 & 0.000 & 0.0 & 0.0 & 0.0 & 0.0 & 0.0 & 0.0 & 0.0 & 0.0 \\
\hline 8.0 & 0.632 & 0.269 & 0.116 & 0.010 & 0.001 & 0.000 & 0.0 & 0.0 & 0.0 & 0.0 & 0.0 & 0.0 & 0.0 & 0.0 \\
\hline 8.5 & 0.594 & 0.234 & 0.072 & 0.006 & 0.0 & 0.0 & 0.0 & 0.0 & 0.0 & 0.0 & 0.0 & 0.0 & 0.0 & 0.0 \\
\hline 9.0 & 0.557 & 0.209 & 0.056 & 0.003 & 0.0 & 0.0 & 0.0 & 0.0 & 0.0 & 0.0 & 0.0 & 0.0 & 0.0 & 0.0 \\
\hline 9.5 & 0.521 & 0.196 & 0.040 & 0.002 & 0.0 & 0.0 & 0.0 & 0.0 & 0.0 & 0.0 & 0.0 & 0.0 & 0.0 & 0.0 \\
\hline 10.0 & 0.479 & 0.162 & 0.022 & 0.001 & 0.0 & 0.0 & 0.0 & 0.0 & 0.0 & 0.0 & 0.0 & 0.0 & 0.0 & 0.0 \\
\hline 10.5 & 0.437 & 0.117 & 0.015 & 0.001 & 0.0 & 0.0 & 0.0 & 0.0 & 0.0 & 0.0 & 0.0 & 0.0 & 0.0 & 0.0 \\
\hline 11.0 & 0.406 & 0.107 & 0.008 & 0.001 & 0.0 & 0.0 & 0.0 & 0.0 & 0.0 & 0.0 & 0.0 & 0.0 & 0.0 & 0.0 \\
\hline 11.5 & 0.375 & 0.127 & 0.006 & 0.000 & 0.0 & 0.0 & 0.0 & 0.0 & 0.0 & 0.0 & 0.0 & 0.0 & 0.0 & 0.0 \\
\hline 12.0 & 0.339 & 0.116 & 0.003 & 0.0 & 0.0 & 0.0 & 0.0 & 0.0 & 0.0 & 0.0 & 0.0 & 0.0 & 0.0 & 0.0 \\
\hline 12.5 & 0.316 & 0.097 & 0.002 & 0.0 & 0.0 & 0.0 & 0.0 & 0.0 & 0.0 & 0.0 & 0.0 & 0.0 & 0.0 & 0.0 \\
\hline 13.0 & 0.278 & 0.075 & 0.001 & 0.0 & 0.0 & 0.0 & 0.0 & 0.0 & 0.0 & 0.0 & 0.0 & 0.0 & 0.0 & 0.0 \\
\hline 13.5 & 0.266 & 0.053 & 0.001 & 0.0 & 0.0 & 0.0 & 0.0 & 0.0 & 0.0 & 0.0 & 0.0 & 0.0 & 0.0 & 0.0 \\
\hline 14.0 & 0.227 & 0.037 & 0.001 & 0.0 & 0.0 & 0.0 & 0.0 & 0.0 & 0.0 & 0.0 & 0.0 & 0.0 & 0.0 & 0.0 \\
\hline 14.5 & 0.219 & 0.026 & 0.001 & 0.0 & 0.0 & 0.0 & 0.0 & 0.0 & 0.0 & 0.0 & 0.0 & 0.0 & 0.0 & 0.0 \\
\hline 15.0 & 0.180 & 0.017 & 0.001 & 0.0 & 0.0 & 0.0 & 0.0 & 0.0 & 0.0 & 0.0 & 0.0 & 0.0 & 0.0 & 0.0 \\
\hline 15.5 & 0.176 & 0.013 & 0.0 & 0.0 & 0.0 & 0.0 & 0.0 & 0.0 & 0.0 & 0.0 & 0.0 & 0.0 & 0.0 & 0.0 \\
\hline 16.0 & 0.170 & 0.011 & 0.0 & 0.0 & 0.0 & 0.0 & 0.0 & 0.0 & 0.0 & 0.0 & 0.0 & 0.0 & 0.0 & 0.0 \\
\hline 16.5 & 0.137 & 0.011 & 0.0 & 0.0 & 0.0 & 0.0 & 0.0 & 0.0 & 0.0 & 0.0 & 0.0 & 0.0 & 0.0 & 0.0 \\
\hline 17.0 & 0.129 & 0.009 & 0.0 & 0.0 & 0.0 & 0.0 & 0.0 & 0.0 & 0.0 & 0.0 & 0.0 & 0.0 & 0.0 & 0.0 \\
\hline 17.5 & 0.099 & 0.0 & 0.0 & 0.0 & 0.0 & 0.0 & 0.0 & 0.0 & 0.0 & 0.0 & 0.0 & 0.0 & 0.0 & 0.0 \\
\hline 18.0 & 0.095 & 0.0 & 0.0 & 0.0 & 0.0 & 0.0 & 0.0 & 0.0 & 0.0 & 0.0 & 0.0 & 0.0 & 0.0 & 0.0 \\
\hline 18.5 & 0.070 & 0.0 & 0.0 & 0.0 & 0.0 & 0.0 & 0.0 & 0.0 & 0.0 & 0.0 & 0.0 & 0.0 & 0.0 & 0.0 \\
\hline 19.0 & 0.065 & 0.0 & 0.0 & 0.0 & 0.0 & 0.0 & 0.0 & 0.0 & 0.0 & 0.0 & 0.0 & 0.0 & 0.0 & 0.0 \\
\hline 19.5 & 0.046 & 0.0 & 0.0 & 0.0 & 0.0 & 0.0 & 0.0 & 0.0 & 0.0 & 0.0 & 0.0 & 0.0 & 0.0 & 0.0 \\
\hline 20.5 & 0.029 & 0.0 & 0.0 & 0.0 & 0.0 & 0.0 & 0.0 & 0.0 & 0.0 & 0.0 & 0.0 & 0.0 & 0.0 & 0.0 \\
\hline 21. & 0.027 & 0.0 & 0.0 & 0.0 & 0.0 & 0.0 & 0.0 & 0.0 & 0.0 & 0.0 & 0.0 & 0.0 & 0.0 & 0.0 \\
\hline 21.5 & 0.025 & 0.0 & 0.0 & 0.0 & 0.0 & 0.0 & 0.0 & 0.0 & 0.0 & 0.0 & 0.0 & 0.0 & 0.0 & 0.0 \\
\hline 22. & 0.017 & 0.0 & 0.0 & 0.0 & 0.0 & 0.0 & 0.0 & 0.0 & 0.0 & 0.0 & 0.0 & 0.0 & 0.0 & 0.0 \\
\hline 22.5 & 0.016 & 0.0 & 0.0 & 0.0 & 0.0 & 0.0 & 0.0 & 0.0 & 0.0 & 0.0 & 0.0 & 0.0 & 0.0 & 0.0 \\
\hline 23. & 0.011 & 0.0 & 0.0 & 0.0 & 0.0 & 0.0 & 0.0 & 0.0 & 0.0 & 0.0 & 0.0 & 0.0 & 0.0 & 0.0 \\
\hline 23.5 & 0.010 & 0.0 & 0.0 & 0.0 & 0.0 & 0.0 & 0.0 & 0.0 & 0.0 & 0.0 & 0.0 & 0.0 & 0.0 & 0.0 \\
\hline 24. & 0.006 & 0.0 & 0.0 & 0.0 & 0.0 & 0.0 & 0.0 & 0.0 & 0.0 & 0.0 & 0.0 & 0.0 & 0.0 & 0.0 \\
\hline 24.5 & 0.006 & 0.0 & 0.0 & 0.0 & 0.0 & 0.0 & 0.0 & 0.0 & 0.0 & 0.0 & 0.0 & 0.0 & 0.0 & 0.0 \\
\hline 25. & 0.004 & 0.0 & 0.0 & 0.0 & 0.0 & 0.0 & 0.0 & 0.0 & 0.0 & 0.0 & 0.0 & 0.0 & 0.0 & 0.0 \\
\hline 26. & 0.002 & 0.0 & 0.0 & 0.0 & 0.0 & 0.0 & 0.0 & 0.0 & 0.0 & 0.0 & 0.0 & 0.0 & 0.0 & 0.0 \\
\hline 27. & 0.002 & 0.0 & 0.0 & 0.0 & 0.0 & 0.0 & 0.0 & 0.0 & 0.0 & 0.0 & 0.0 & 0.0 & 0.0 & 0.0 \\
\hline 28. & 0.002 & 0.0 & 0.0 & 0.0 & 0.0 & 0.0 & 0.0 & 0.0 & 0.0 & 0.0 & 0.0 & 0.0 & 0.0 & 0.0 \\
\hline 29. & 0.001 & 0.0 & 0.0 & 0.0 & 0.0 & 0.0 & 0.0 & 0.0 & 0.0 & 0.0 & 0.0 & 0.0 & 0.0 & 0.0 \\
\hline 30.0 & 0.001 & 0.0 & 0.0 & 0.0 & 0.0 & 0.0 & 0.0 & 0.0 & 0.0 & 0.0 & 0.0 & 0.0 & 0.0 & 0.0 \\
\hline
\end{tabular}

